\title{
On the properties of the Dickey-Pantula test against fractional alternatives
}

\author{
Juan J. Dolado ${ }^{\mathrm{a}, *}$, Francesc Marmol ${ }^{\mathrm{b}}$ \\ ${ }^{a}$ Departamento de Economia, Universidad Carlos III de Madrid, 28903 Getafe, Spain \\ ${ }^{\mathrm{b}}$ Departamento de Estadistica y Econometria, Universidad Carlos III de Madrid, 28903 Getafe, Spain
}

Received 4 February 1997; accepted 2 June 1997

\begin{abstract}
The limit properties of the testing sequence underlying the Dickey-Pantula test for a double unit root in a time series are derived when the true data generating process is assumed to be nonstationary fractionally integrated. (C) 1997 Elsevier
\end{abstract} Science S.A.

Keywords: Dickey-Pantula test; Nonstationary fractionally integrated processes

JEL classification: $\mathrm{C} 12 ; \mathrm{C} 15 ; \mathrm{C} 22$

\section{Introduction}

Although the stochastic behaviour of many economic time series has been reported in the literature to be well approximated by integrated processes of order one, denoted $I(1)$, there are some series, especially nominal ones (e.g., money holdings, prices, wages, etc.) which appear to be potentially better described as $I(2)$ processes. Considering the problem of testing for a double unit root, Dickey and Pantula (1987) and Pantula (1989) have suggested a popular sequential testing procedure in applied work which takes the largest number of unit roots under consideration as the first maintained hypothesis and then decreases the order of differencing each time the current null hypothesis is rejected.

In general, $I(1)$ and $I(2)$ processes can be considered as particular cases of the more general family of fractionally integrated processes, denoted $\operatorname{FI}(d)$. As is well-known, a stochastic process $y_{i}$ is called $\mathrm{FI}(d)$ if $\Delta^{d} y_{t} \sim I(0)$, where $d$ is allowed to be a real number rather than just an integer one. When $d \geq \frac{1}{2}$, the series is nonstationary, denoted $\operatorname{NFI}(d)$. Sowell (1990) derived the limit distribution of the standard Dickey-Fuller (DF) for a single unit root, based upon the $t$-statistic when the true process is $\operatorname{NFI}(d)$ with $d \in\left(\frac{1}{2}, \frac{3}{2}\right)$, showing that it diverges to $-\infty(+\infty)$ when $d<1(d>1)$. Hence, the $t$-test is consistent for $d \in\left(\frac{1}{2}, 1\right)$ and has zero power for $d \in\left(1, \frac{3}{2}\right)$.

*Corresponding author. Tel.: +34 1 6249300; fax: +34 1 6249313; e-mail: dolado@eco.uc3m.es 
In this paper we extend the previous arguments to the sequential testing procedure advocated by Dickey and Pantula (DP), allowing the analysis to cover the case of $I(2)$ processes. We find that Sowell's results can be generalized to these higher integrated processes. In fact, the properties of the testing sequence are derived for all values of $d$ within the nonstationary range and thus can be extended to testing for three or more unit roots in the presence of $\operatorname{NFI}(d)$ alternatives. Sketches of the proofs are relegated to Appendix A.

\section{The model and the Dickey-Pantula test}

Throughout the paper, we shall assume that the true data generating process (DGP) of $y_{t}$ is the following NFI process

$$
\Delta^{d} y_{t}=\varepsilon_{t}
$$

where $d \geq \frac{1}{2}, \varepsilon_{t} \sim \operatorname{iid}\left(0, \delta^{2}\right)$ and $y_{0}=0$ for $t \leq 0$. Noticing that $d$ can always be decomposed as $d=\alpha+\delta$, where $\alpha=1,2,3, \ldots$ and $|\delta|<\frac{1}{2}$, (1) can be reparameterized as

$$
\Delta^{a} y_{t}=\eta_{t}, \Delta^{\delta} \eta_{t}=\varepsilon_{t},
$$

that is, any $\operatorname{NFI}(d)$ process can be expressed as an integer $I(\alpha)$ process with stationary and ergodic fractionally integrated $\operatorname{SFI}(\delta)$ innovation.

Let $\sigma_{\eta T}^{2}=\operatorname{var}\left(S_{T}\right)$, where $S_{t}=\Sigma_{j=1}^{t} \eta_{j}$. The growth rate of this partial sums' variance was proved by Sowell (1990) to be equal to

$$
T^{-1-2 \delta} \sigma_{\eta T}^{2} \stackrel{p}{\rightarrow} \frac{\sigma^{2} \Gamma(1-2 \delta)}{(1+2 \delta) \Gamma(1+\delta) \Gamma(1-\delta)} \equiv \theta_{\eta}^{2},
$$

say, where $\Gamma(\cdot)$ denotes the gamma or generalized factorial function. Furthermore, under the additional assumption that $\varepsilon_{t}$ verifies $E\left|\varepsilon_{t}\right|^{g}<\infty$ for $g \geq \max \{4,-8 \delta / 1+2 \delta\}$, the following functional central limit theorem applies to this type of process:

$$
\sigma_{\eta T}^{-1} S_{[\mathrm{Tr}]} \Rightarrow \frac{1}{\Gamma(1+\delta)} \int_{0}^{r}(r-s)^{\delta} \mathrm{d} W(s), \quad\left(\equiv W_{\delta}(r)\right),
$$

where $W(r)$ is a standard Brownian motion on [0,1] associated with the $\varepsilon_{t}$ sequence and the symbols " $\Rightarrow$ " and " $\rightarrow$ " denote weak covergence and convergence in probability, respectively.

Consider now the two steps involved in the DP sequential tests. In the first stage, the $t$-ratio of $\hat{\beta}_{1}$ in the following regression

$$
\Delta^{2} y_{t}=\hat{\beta}_{1} \Delta y_{t-1}+\text { res. },
$$

is compared with the corresponding DF critical value in a one-sided lower-tail test in order to test the null hypothesis of two unit roots $\left(y_{t} \sim I(2)\right)$ against the alternative of a single unit root $\left(y_{t} \sim I(1)\right)$. Then, the following theorem holds. 
Theorem 1. Under DGP(2), the t-test of $\hat{\beta}_{1}$ in Eq. (5) verifies that

(i) if $0.5 \leq d<1.5, t_{\beta_{1}}=O_{p}\left(T^{1 / 2}\right)$ and $t_{\beta_{1}} \stackrel{p}{\rightarrow}-\infty$,

(ii) if $1.5 \leq d<2, t_{\beta_{1}}=O_{p}\left(T^{2-d}\right)$ and $t_{\beta_{1}} \stackrel{p}{\rightarrow}-\infty$,

(iii) if $d=2, t_{\beta_{1}}=O_{p}(1)$,

(iv) if $2<d<2.5, t_{\beta_{1}}=O_{p}\left(T^{\mathrm{d}-2}\right)$ and $t_{\beta_{1}} \stackrel{p}{\rightarrow}$, and

(v) if $d \geq 2.5, t_{\beta_{1}}=O_{p}\left(T^{1 / 2}\right)$ and $t_{\beta_{1}} \stackrel{p}{\rightarrow} \infty$.

As expected, these properties mimic those obtained by Sowell (1990) in the test of the null of a single unit root versus the alternative of stationarity. Thus, the $t$-ratio only has a well-defined asymptotic distribution when $d=2$, it is a consistent test for $d<2$ and has zero power when $d>2$.

Next, if the null hypothesis above is rejected, the second stage in the DP procedure proceeds to test the null of $y_{t} \sim I(1)$ against the alternative of $y_{t} \sim I(0)$ computing the $t$-ratio of $\hat{\beta}_{2}$ in the regression model

$$
\Delta^{2} y_{t}=\hat{\beta}_{1} \Delta y_{t-1}+\hat{\beta}_{2} y_{t-1}+\text { res. },
$$

and comparing it with the DF critical value in a one-sided test. In this case, the following theorem applies.

Theorem 2. Under DGP (2), the t-test of $\hat{\beta}_{2}$ in (6) verifies that

(i) if $0.5 \leq d<1, t_{\beta_{2}}=O_{p}\left(T^{1-d}\right)$ and $t_{\beta_{2}} \stackrel{p}{\rightarrow}-\infty$,

(ii) if $d=1, t_{\beta_{2}}=O_{p}(1)$,

(iii) if $1<d<1.5, t_{\beta_{2}}=O_{p}\left(T^{d-1}\right)$ and $t_{\beta_{2}} \stackrel{p}{\rightarrow}$, and

(v) if $d \geq 1.5, t_{\beta_{2}}=O_{p}\left(T^{1 / 2}\right)$ and $t_{\beta_{2}} \stackrel{p}{\rightarrow} \infty$.

The most remarkable feature of this result is that it mimics the findings in Theorem 1 . The relevant $t$-statistic has only a well-defined limiting distribution when $d=1$. If $d<1$, the test will be consistent and if $d>1$, it will have zero power. Finally, if $1<d<2$, the sequential testing procedure will classify the process as an $I(1)$ one. For instance, if $d=1.8$, then, following Theorem 1, the null hypothesis of two unit roots is asymptotically rejected in a one-sided test whereas, following Theorem 2 , the null of a single unit root is not rejected. Consequently, the DP test classifies a NFI(1.8) as an I(1) process as the sample size gets sufficiently large.

To check these results, we generated such a process based on 5000 replications with $T=100$ and $T=250$ observations, with standard Gaussian innovations. For $T=100$ and considering one-sided tests with level 0.05 , , the percentage of rejections in the first stage of the DP procedure is $39.3 \%$ whilst the corresponding proportion in the second stage is $17.7 \%$. For $T=250$, the rejection rates are $68.1 \%$ and $4.3 \%$, respectively. In line with the Monte Carlo evidence reported in Diebold and Rudebusch (1991)

${ }^{1}$ All computations were done in $S$-plus. 
and Hassler and Wolters (1994), the previous results point out that asymptotic considerations can be severely misleading in finite samples, since the power of unit root tests can be very low within the Euclidean interval $|d-1 / 2|$ of the corresponding null hypothesis. As Sowell (1990) conjectures, this is because the limit distributions of the $t$-statistics depend upon two underlying random variables (expressions (7)-(9) in Appendix A) with a slow rate of convergence to its asymptotic distribution for a plausible range of $d$ values.

\section{Concluding remarks}

Theorems 1 and 2 characterize the asymptotic behaviour of the DP testing sequence under fractional alternatives, extending previous results on the properties of DF unit root tests.

Our basic conclusion is that mechanical application of the DP procedure can lead to misleading results. Moreover, this conclusion can be extended to other testing approaches within the same family, such as those proposed by Hasza and Fuller (1979), Sen and Dickey (1987) and Haldrup (1994).

Finally, although, for the sake of brevity, we have confined the results to a maximum of two unit roots, the above findings can be easily generalized to the more general sequential procedure consisting of testing a null hypothesis of $k$ unit roots against an alternative of $k-1$ unit roots. Namely, as $T \uparrow \infty$ the sequence will stop when the true $d$ verifies the inequality $k-1<d<k$.

\section{Appendix A}

Since the proofs of the results are lengthy in detail we shall only report their main steps and the relevant rates of convergence. Full detailed derivations are available from the authors upon request.

Proof of Theorem 1. Under model (5), the least squares slope estimator and its corresponding $t$-ratio have the following expressions:

$$
\hat{\beta}_{1}=\frac{\sum\left(\Delta y_{t-1}\right)\left(\Delta^{2} y_{t}\right)}{\sum\left(\Delta y_{t-1}\right)^{2}},
$$

and

$$
t_{\beta_{1}}=\frac{\sum\left(\Delta y_{t-1}\right)\left(\Delta^{2} y_{t}\right)}{\hat{\sigma}\left[\sum\left(\Delta y_{t-1}\right)^{2}\right]^{1 / 2}},
$$

where $\hat{\sigma}^{2}=T^{-1} \Sigma\left(\Delta^{2} y_{t}-\hat{\beta}_{1} \Delta y_{t-1}\right)^{2}$ and with the symbol $\sum$ denoting $\sum_{t=1}^{T}$. When $d \in\left[\frac{3}{2}, \frac{5}{2}\right), d=2+\delta$ and $\Delta y_{t} \sim \operatorname{NFI}(d-1)$. Consequently, using (3), (4) and the continuous mapping theorem (CMT), we have that $\Sigma\left(\Delta y_{t-1}\right)^{2}=O_{p}\left(T^{2 d-2}\right)$. In the same manner, it is rather direct to prove that $\Sigma\left(\Delta y_{t-1}\right)\left(\Delta^{2} y_{t}\right)=$ $\frac{1}{2}\left(\Delta y_{T}\right)^{2}-\frac{1}{2} \sum \eta_{t}^{2}$. Hence, $\theta_{\eta}^{2}=\sigma^{2}, W_{\delta}(r)=W(r)$ and

$$
T^{-1} \sum\left(\Delta y_{t-1}\right)\left(\Delta^{2} y_{t}\right) \Rightarrow \frac{1}{2} \sigma^{2}[W(1)]^{2}-\frac{1}{2} \sigma^{2},
$$


when $d=2$,

$$
T^{1-2 \delta} \sum\left(\Delta y_{t-1}\right)\left(\Delta^{2} y_{t}\right) \Rightarrow \frac{1}{2} \theta_{\eta}^{2}\left[W_{\delta}(1)\right]^{2},
$$

when $2<d<\frac{5}{2}$, and

$$
T^{-1} \sum\left(\Delta y_{t-1}\right)\left(\Delta^{2} y_{t}\right) \stackrel{p}{\rightarrow}-\frac{\sigma^{2} \Gamma(1-2 \delta)}{2 \Gamma^{2}(1-\delta)}
$$

when $\frac{3}{2} \leq d<2$, meaning that, when $d \geq 2 \hat{\beta}_{1}=O_{p}\left(T^{-1}\right)$, whereas $\hat{\beta}_{1}=O_{p}\left(T^{3-2 d}\right)$ in the case where $d<2$.

With regard to the $t$-Student statistic, first notice that it is straightforward to prove that $\hat{\sigma}^{2} \stackrel{p}{\rightarrow}$ $\operatorname{var}\left(\eta_{t}\right)$ for all $\delta \in\left(-\frac{1}{2}, \frac{1}{2}\right)$, from which it follows that $t_{\beta_{1}}=O_{p}(1)$ when $d=2, t_{\beta_{1}}=O_{p}\left(T^{d-2}\right)$ if $d>2$ such that $t_{\beta_{1}} \stackrel{p}{\rightarrow} \infty$ given that (A.2) is a positive random variable, while $t_{\beta_{1}}=O_{p}\left(T^{2-d}\right)$ with $t_{\beta_{1}} \stackrel{p}{\rightarrow}-\infty$ if $d<2$, since (A.3) is negative.

When $d \geq \frac{5}{2}$, then $\alpha \geq 3, \Delta y_{t} \sim \operatorname{NFI}(d-1)$ and $\Delta^{2} y_{t} \sim \operatorname{NFI}(d-2)$. In this case, using (3), (4) and the CMT, it can be proved that $T^{1-\alpha} \sigma_{\eta T}^{-1} S_{[\mathrm{Tr}]} \Rightarrow W_{\delta}^{\alpha}(r), \alpha \geq 1$. Hence, some manipulation yields $\sum\left(\Delta y_{t-1}\right)^{2}=O_{p}\left(T^{2 d-2}\right)$ and $\sum\left(\Delta y_{t-1}\right)\left(\Delta^{2} y_{t}\right)=O_{p}\left(T^{2 d-3}\right)$, meaning that $\hat{\beta}_{1}=O_{p}\left(T^{-1}\right), \hat{\sigma}^{2}=O_{p}\left(T^{2 d-5}\right)$ and $t_{\beta_{1}}=O_{p}\left(T^{1 / 2}\right)$ with $t_{\beta_{1}} \stackrel{p}{\rightarrow} \infty$.

Finally, when $d \in\left[\frac{1}{2}, \frac{3}{2}\right), \Delta y_{t}=\eta_{t} \sim \operatorname{SFI}(\delta)$ so that $\Delta^{2} y_{t}$ becomes a non-invertible (but stationary) fractionally integrated process. In this case, it is straightforward to prove that $\hat{\beta}_{1}=O_{p}(1), \hat{\sigma}^{2}=O_{p}(1)$ and $t_{\beta_{1}}=O_{p}\left(T^{1 / 2}\right)$ with $t_{\beta_{1}} \stackrel{p}{\rightarrow}-\infty$

Proof of Theorem 2. Under model (6), the least squares slope estimate of $y_{t-1}$ and its corresponding $t$-ratio have the following expressions:

$$
\hat{\beta}_{2}=\frac{\left[\sum y_{t-1} \Delta^{2} y_{t}\right]\left[\sum\left(\Delta y_{t-1}\right)^{2}\right]-\left[\sum y_{t-1} \Delta y_{t-1}\right]\left[\sum \Delta y_{t-1} \Delta^{2} y_{t}\right]}{\left[\sum y_{t-1}^{2}\right]\left[\sum\left(\Delta y_{t-1}\right)^{2}\right]-\left[\sum y_{t-1} \Delta y_{t-1}\right]^{2}},
$$

and

$$
t_{\beta_{2}}=\frac{\hat{\beta}_{2}}{\hat{\sigma}_{\beta_{2}}}
$$

with

$$
\hat{\sigma}_{\beta_{2}^{2}}=\hat{\sigma}^{2} \frac{\left[\sum\left(\Delta y_{t-1}\right)^{2}\right]}{\left[\sum y_{t-1}^{2}\right]\left[\sum\left(\Delta y_{t-1}\right)^{2}\right]-\left[\sum y_{t-1} \Delta y_{t-1}\right]^{2}} .
$$

Now, proceeding as in Theorem 1, it can be proved that, when $d \geq \frac{5}{2}, \sum y_{t-1}^{2}=O_{p}\left(T^{2^{d}}\right)$, $\sum y_{t-1} \Delta y_{t-1}=O_{p}\left(T^{2 d-1}\right)$ and $\sum y_{t-1} \Delta^{2} y_{t}\left(T^{2 d-1}\right)$, meaning that $\hat{\beta}_{2}=O_{p}\left(T^{-1}\right)$ and $\hat{\beta}_{1}=O_{p}(1)$. Hence, given that $\hat{\sigma}^{2}=T^{-1} \sum\left(\Delta^{2} y_{t}-\hat{\beta}_{1} \Delta y_{t-1}-\hat{\beta}_{2} y_{t-1}\right)^{2}$, it follows that $\hat{\sigma}^{2}=O_{p}\left(T^{2 d-3}\right)$ which, in turn, implies that $\hat{\sigma}_{\beta_{2}}^{2}=O_{p}\left(T^{-3}\right)$, and hence, $t_{\beta_{2}}=O_{p}\left(T^{1 / 2}\right)$ such that $t_{\beta_{2}} \stackrel{p}{\rightarrow}$. 
When $d \in\left[\frac{3}{2}, \frac{5}{2}\right), \Delta^{2} y_{t} \sim \operatorname{SFI}(\delta)$ and then $\sum y_{t-1} \eta_{t}=O_{p}\left(T^{2 d-2}\right)$. Consequently, it is direct to prove that $\hat{\beta}_{2}=O_{p}\left(T^{-2}\right), \hat{\sigma}^{2}=O_{p}\left(T^{2 d-3}\right), \hat{\sigma}_{\beta_{2}}=O_{p}\left(T^{-3}\right)$ and $t_{\beta_{2}}=O_{p}\left(T^{1 / 2}\right)$ such that $t_{\beta_{2}} \stackrel{p}{\rightarrow}$.

Lastly, in the case where $d \in\left[\frac{1}{2}, \frac{3}{2}\right), \Delta y_{t} \sim \operatorname{SFI}(d-1)$. Therefore, after some direct but tedious calculus it can be deduced that when $d=1, \hat{\beta}_{2}=O_{p}\left(T^{-1}\right), \hat{\beta}_{1}=O_{p}(1), \hat{\sigma}^{2}=O_{p}(1)$ and $t_{\beta_{2}}=O_{p}(1)$, whereas if $1<d<\frac{3}{2}, \hat{\beta}_{2}=O_{p}\left(T^{-1}\right), \hat{\beta}_{1}=O_{p}(1), \hat{\sigma}^{2}=O_{p}(1)$ and $t_{\beta_{2}}=O_{p}\left(T^{d-1}\right)$ with $t_{\beta_{2}} \stackrel{p}{\rightarrow} \rightarrow$. Conversely, when $\frac{1}{2} \leq d<1, \hat{\beta}_{1}=O_{p}(1), \hat{\sigma}^{2}=O_{p}(1)$ and $\hat{\beta}_{2}=O_{p}\left(T^{1-2 d}\right)$ with nonpositive support so that $t_{\beta_{2}}=O_{p}\left(T^{1-d}\right)$ and $t_{\beta_{2}} \stackrel{p}{\rightarrow}-\infty$. This last statement completes the proof of the theorem.

\section{References}

Dickey, D., Pantula, S., 1987. Determining the order of differencing in autoregressive processes. Journal of Business and Economic Statistics 15, 455-461.

Diebold, F., Rudebusch, G., 1991. On the power of Dickey-Fuller tests against fractional alternatives. Economics Letters 35 , 155-160.

Haldrup, N., 1994. Semiparametric tests for double unit roots. Journal of Business and Economic Statistics 12, $109-122$.

Hassler, U., Wolters, J., 1994. On the power of unit root tests against fractional alternatives. Economics Letters 45, 1-5.

Hasza, D., Fuller, W., 1979. Estimation of autoregressive processes with unit roots. Annals of Statistics 7, 1106-1120.

Pantula, S., 1989. Testing for unit roots in time series data. Econometric Theory 5, 256-271.

Sen, D., Dickey, D., 1987. Symmetric test for second differencing in univariate time series. Journal of Business and Economic Statistics 5, 463-473.

Sowell, F., 1990. The fractional unit root distribution. Econometrica 58, 495-505. 